\title{
Applying Human-Centric Functional Modeling and General Collective Intelligence to the Process of Observation
}

\section{Keywords}

Human-Centric Functional Modeling, cognition, experimental observation, existential philosophy

\begin{abstract}
Eastern existential traditions focus on practices meant to increase one's ability to observe what is true in one's awareness of this existence. The accuracy of surveys depends on the ability of the respondents to observe truth. Practices that increase one's ability to observe what is true are formalized in this paper through the methodology of Human-Centric Functional Modeling, so they can be used to pre-qualify survey respondents according to their ability to understand principles of truthful observation. These principles are namely, that truth is what is true within one's own experience (what one can observe directly or prove through "first principles", that is prove from such direct observations). Truth is not what is within one's beliefs, with the exception of what in one's own observation those beliefs function to achieve. Nor is truth what any given academic tradition might assume, unless those assumption can be observed directly or deduced from first principles. In addition, this paper explores why the use of General Collective Intelligence or GCI is required to remove barriers to the complexity of observations that can be truthfully made. The justifications for these conclusions is presented, as is a methodology for using these conclusions to continuously and adaptively improve survey results.
\end{abstract}

\section{Introduction}

For thousands of years, through self-observation alone, without any external diagnostic equipment, yogis and other practitioners of Eastern existential traditions have been developing an understanding of the functions of this profoundly complex human system and the even more complex universe around it, and have been demonstrating the truth of that understanding through their ability to use it to reliably achieve an outcome, in their case, the outcome of well-being. Given the difficulty modern academics have in achieving convergence in understanding over only a few disciplines, despite our modern ability to store and disseminate information, the fact that these traditions could converge on any single understanding at all, much less an understanding of the universe, where that understanding actually functions to achieve some outcome, is stunning. A fundamentally different process of understanding must be at work than in the modern academic tradition.

With this inspiration to search for it, a process attempting to replicate this fundamentally different process of understanding was defined. Recently formalized in a methodology called Human-Centric Functional Modeling (HCFM), this process models how the human system and the world around it function, in order to more reliably navigate that understanding. HCFM uses a model of human cognition in the attemp to to increase an individual's capacity to navigate their own human cognition between any two sets of concepts, so that it is easier to navigate towards the definition of any problem their human cognition has (i.e. they have) the capacity to define, and towards any solution their human cognition has the capacity to discover. Using HCFM might increase an individual's capacity to navigate their cognition towards an understanding. However the cognitive model associated with HCFM suggests there is still a well-defined limit to the complexity of problems or solutions that can reliably be conceived by any individual cognition, and that without a system of group decision-making with very specific properties, there is a limit to the degree that cooperation between individuals can reliably scaled to increase this capacity for complexity.

To have the capacity to understand how yogis can reliably converge on an understanding within this limit of cognition, and therefore to have the capacity to understand the value of Human-Centric Functional Modeling, it's important to first be able to understand and observe a few things in one's own thought. Psychologists have identified two types of reasoning, rational methodical reasoning, and 
intuitive reasoning. Rational methodical reasoning is like an equation. It has specific inputs, and a welldefined process that produces specific outputs. Intuitive reasoning is a black box that produces answers by detecting past patterns. It's important to recognize that while we may think we are reasonable people, all rational methodical reasoning must rely on intuitive reasoning at some point, in particular where the facts, or where any reasoning required to evaluate those facts in any chain of our reasoning processes are unavailable. Since the lengths of our reasoning processes and the subjects that our reasoning might explore are unbounded, while the facts and reasoning at our disposal are bounded, we must always at some point reach reasoning that we can't evaluate methodically. We must then rely on our intuition to tell us what is likely true. For example, in the statement "the leaf is green", the observer assumes that the term "the" indicating an instance of something, the term "leaf", the term "is" indicating a state of existence, and the term "green" indicating a color all have rational explanations. One might be able to come up with all these rational explanations, but all the terms in those rational explanations must also have rational explanations if reasoning is to be completely rational. It is easy to see that the number of terms requiring rational explanations quickly explodes, to the point at which the answer is "it just is". Or in other words, to the point at which the answer must be obtained through intuitive reasoning,

The reasoning style of using functional models to navigate one's understanding that yogi's employ, can be consciously executed in one's rational processes, and if intuition depends on patterns of past solutions, might also be programmed into one's so-called black box of intuition through observing the validity of functional modeling in multiple instances. This use of functional modeling is essentially defining truth as what we can within our own awareness observe any entity we can conceive of as functioning to achieve, rather than defining truth in terms of a theory someone else has proved, or belief someone else has given us. What is true for a yogi or any other practitioner of any existential philosophy is what is within his experience of this existence. Existential philosophies define practices that equate to repeated experiments testing one's ability to accurately observe one's experience when using different processes of awareness or understanding, and in doing so to hone one's awareness and understanding.

If truth is what in our awareness and understanding reliably functions to achieve an outcome, then being aware of and understanding this simple distinction between truth and falsehood enables our understanding to reliably converge on truth simply by continually expanding our observation until reliability can be determined. The importance of this distinction is that it can be generalized and used to refine all general categories of observation to be more truthful. Truth, for example, is not quantum theory, and truth is not general relativity, truth is that these theories function very accurately to make predictions in time scales and energies we can conceive of. Failing to understand that quantum theory or any other theory is not "truth" leads to scientists treating theories as dogma they adhere to, rather than being free of such entanglements and instead just adhering to science.

\section{Modeling the Conceptual Space}

From the perspective of HCFM, any system is a conceptual entity that is dynamic in that it changes state. In the HCFM approach, dynamic entities are described in terms of function, specifically the minimal set of observed functions by which they change state, and from which all other observed functions can be composed. This process of observation is human-centric because it is limited to observations that can be made within innate human perception rather than being in terms of constructs such as beliefs or intellectual theories about the mechanisms by which those functions are implemented, or in any other terms that can't yet be validated within observation.

Dynamic entities (systems) are not only described in terms of the minimal set of human-observable functions capable of describing all their behaviors, but in addition are described in terms of the states accessible through these functions. The set of these states forms a "functional state space" through 
which the system moves. A Functional Modeling Framework (FMF) [7] developed using this approach has been used to define a model for cognition as an adaptive problem-solving process through which each human cognition adapts to solve problems posed by its internal and external environment. In the FMF all concepts accessible to a system of cognition are represented as forming a "conceptual space". This conceptual space is the functional state space of the cognitive system. The cognitive system navigates between concepts using reasoning processes. In this simple functional model, any process of observation or any other reasoning process is also defines as having a simple functional model with concepts as inputs, and outputs, and with concepts forming the context of execution.

\section{Defining Accuracy of Observation in Conceptual Space}

An observer's ability to observe also depends on one simple understanding. In functional terms, an observation is a solution to the problem of categorizing what was observed, a problem is a gap between two sets of concepts, and a solution is a reasoning process that bridges those two sets of concepts, or in other words a reasoning process with the initial set of concepts as inputs, the final set as outputs, and with other concepts forming the context of execution. By expanding the category of any input, output, context, or reasoning involved, we can make our reasoning apply to a much broader set of concepts. By gaining sufficient capacity to expand such conceptual categories, we can generalize any problem definition until it becomes reliably solvable with an existing reasoning solution. Or we can reliably generalize existing reasoning until it solves any particular problem.

This model of human cognition as navigating a conceptual space in which concepts might be resolved at different degrees of precision suggests that generalization is an important part of being able to reliably arrive at an observation. Just like a bullet can't reliably hit a target that's too far off in the distance to resolve, human cognition can't reliably define or solve problems at a specific level (i.e. can't hit the target with a small bullet) when those problems are of higher order complexity than humans are capable of. In order to be reliably able to solve those higher-order complexity problems, human must have the capacity to reliably increase the degree of abstraction (generalization) with which they define problems or solutions until defining both are reliably achievable. In other words they need to increase the size of the bullet until the target can reliably be hit even if they can't see it clearly. The catch of course is that the larger the bullet, the less likely one is to have everything required to fire it.

In academia one is forced to reason in terms of the ideas of others in far greater detail than can be justified within one's own experience. This increases the complexity of the problem of observation until it is not reliably solvable. If so it is no mistake that all academia has not converged into a single understanding across a wide range of subjects. However, in mathematics and philosophy, proofs or arguments made from "first principles" are those that are self-contained and don't need to refer to others. In order to be able to observe truth within our experience one must expand one's ability to observe by first principles through being able to generalize until one can identify truth, as opposed to simply avoiding falsehood. When one focuses on untruth, by for example trying to observe by focusing on any specific point that one believes one can disprove through assumptions or beliefs outside of one's own observation, then it might become impossible to expand perspectives with any observation of truth. When one instead focuses on truth, through more broadly generalizing until one can observe what appears to be truthful, then old perspective might be expanded to include truth. Whether these processes actually function to increase capacity to observe truth remains to be confirmed experimentally.

In any case, one might expect that developing the ability to reliably separate awareness of truth from opinion, might enable one to objectively test that awareness on every perspective, even those one might be inclined to profoundly disagree with, so that eventually one's inclination to label with like or dislike eventually might disappear and one can just observe things as they are. When as observer has removed 
the limitation to their ability to observe, and when they have developed the capacity to reliably discern truth, as they expand their observation it might then reliably converge on truth.

\section{Observability of Problems and Solutions}

Because HCFM represents all the concepts involved in problems and solutions as forming a "conceptual space", and because in HCFM the reasoning involved in problem-solving navigates from one set of concepts to another, and therefore traces a path through conceptual space, adaptive problem solving has a visual representation that gives observability a well-defined interpretation.

A problem is a gap between two concepts, that is, a gap between points in conceptual space. In order to have the capacity to reliably observe a problem, one must have the capacity to locate those two points in conceptual space. In order to reliably locate those points in conceptual space, one must have the ability to generalize the problem until the problem is reliably observable. The process of generalization defines the problem in terms of a gap between two larger categories of concepts. One might not be able to understand the difference between two very specific concepts, like an up quark and a down quark, but one can reliably understand the difference between two sufficiently general concepts, like a thing, and a thing that differs from that thing by one or more properties.

\section{Visual Representation of Generalizing a Problem Definition within Conceptual Space}
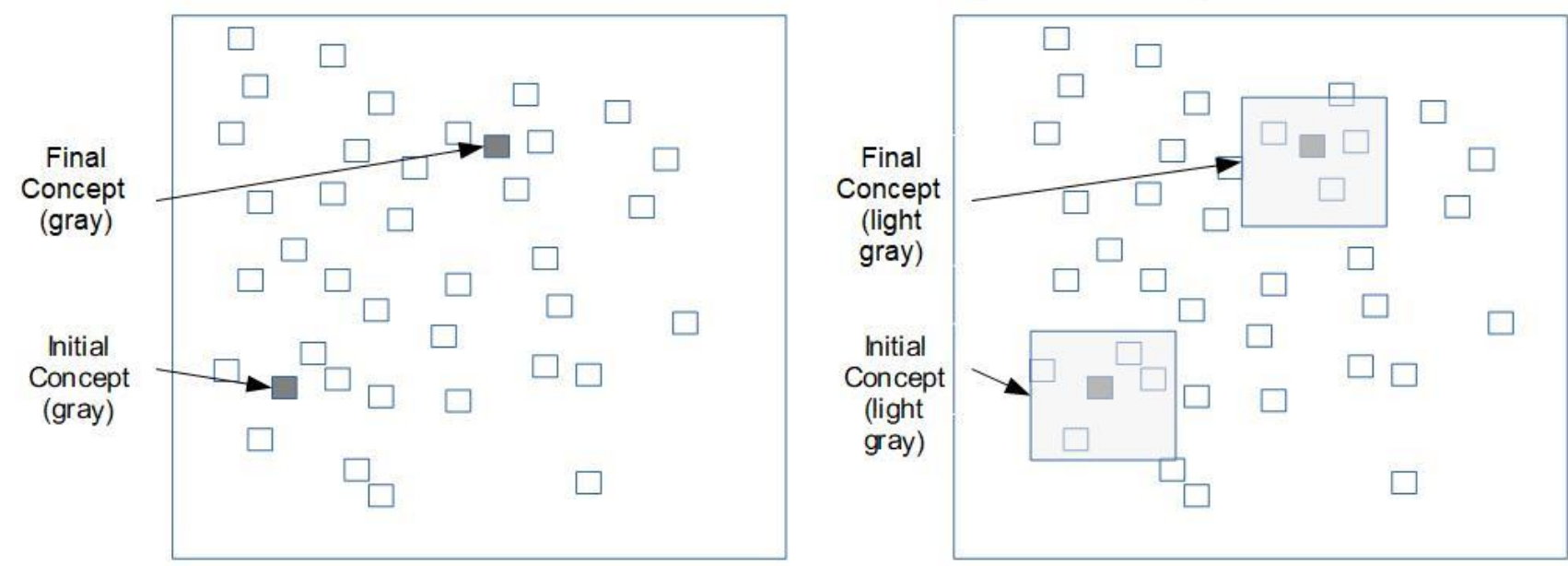

Figure 1: A problem definition is a gap (left) between two specific concepts in conceptual space. Generalization defines the problem to be a gap (right) between two larger categories of concepts.

Or generalization is a matter of of generalizing an existing solution until it solves a new problem. 


\section{Visual Representation of Generalizing a Solution within Conceptual Space}
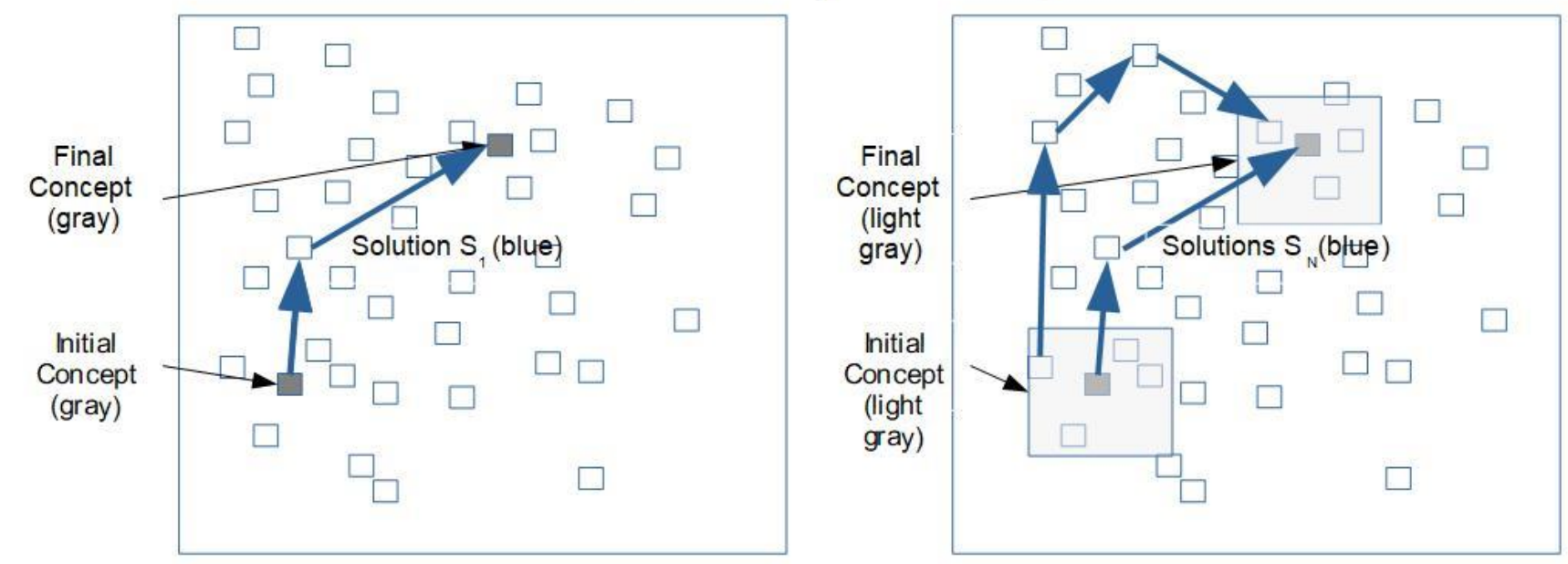

Figure 2: A solution is a path (left) between two specific concepts in conceptual space. Generalization defines the solution to be any path (right) between two larger categories of concepts.

Assumptions or beliefs can be observed to sometimes be untruthful. Assumptions such as arguments derived from references rather than direct observations or arguments defined according to first principles, or beliefs adopted from others, might reliably converge on truth only in the restricted set of circumstances in which assumptions are reliably truthful. If truth is awareness of the location of a concept in conceptual space, that location must be defined in terms of it's position relative to another concept (another point) in conceptual space. That relative position is a process of reasoning or observation. Attempting to locate any concept in conceptual space from any concepts representing assumptions or adopted beliefs is not reliably achievable if neither assumptions nor beliefs reliably converge on truth in all circumstances.

In addition, if one does not understand the intellectual tradition, or does not understand the belief system, one also might not have the ability to observe. Since intellectual reasoning (arguing using references rather than arguing according to first principles) is based on assumed truths, and since beliefs are based on adopted assumptions of truths, definitions of problems or solutions can not be reliably located in conceptual space, and therefore cannot be reliably observable through intellectual arguments or any belief systems since both intellectual traditions and belief systems can potentially differ.

Finally, creating the capacity to reliably observe a problem that is too high in complexity to currently be resolvable, requires increasing capacity for complexity through a process such as scaling cooperation to achieve problem-solving over larger and larger groups, while also decoupling parts of the problem, to reliably increase the number of interactions between functions of a system that can be conceived by the group, until the required capacity for observing complexity is achieved. 


\section{Cognitive Complexity}
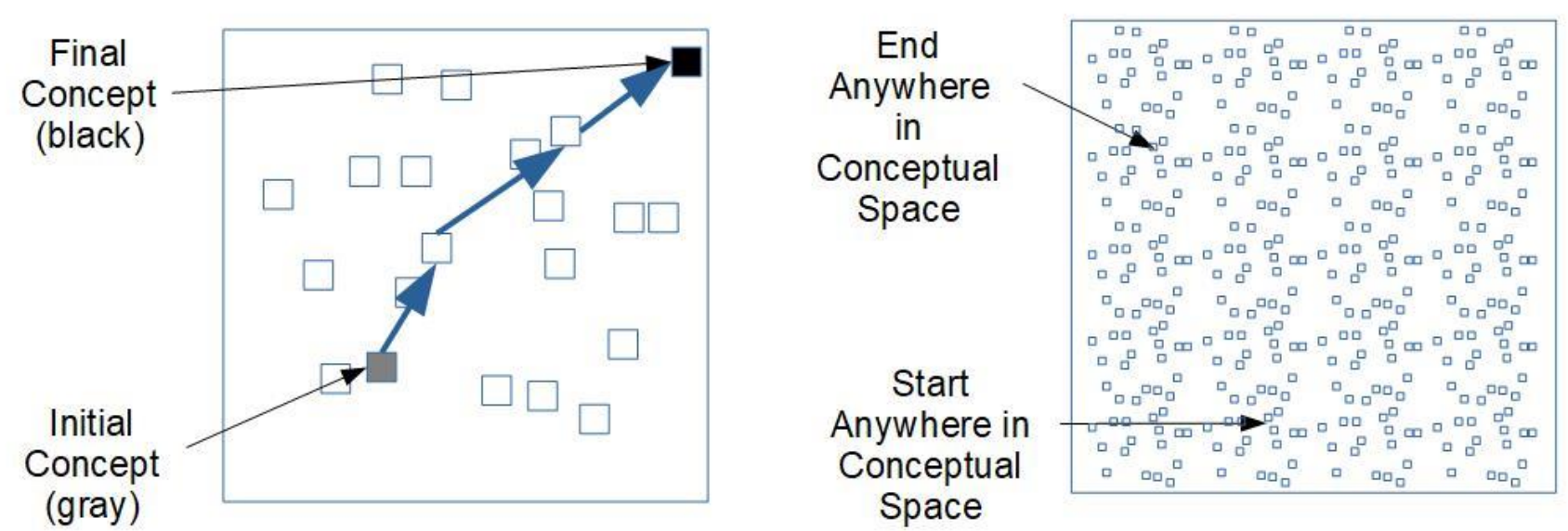

Figure 3: Problems with low cognitve complexity (left) navigate simpler paths between concepts resolved at lower resolution (larger). Problems with high cognitive complexity (right) navigate more complex paths between concepts resolved at higher resolution (smaller), and potentially do so at a much higher level of abstraction so they might start and end anywhere in conceptual space. Without GCI to create the required cognitive capacity to conceive such problem definitions or their solutions, such solutions might not even be recognized as solving the same problem, much less be recognized as being far better solutions.

\section{Pre-Qualification of Survey Respondents}

The criteria that any observation process must have in order to be consistent with the HCFM methodology are:

\section{Features of a Human-Centric Functional Modeling Based System of Observation}

Truth or falsehood observed regarding a system is defined in terms of a function of the system that can be observed, not any beliefs, and not any assumptions, regardless of whether those assumptions have academic references.

The observer is provided specific instruction in each process of observation that gives them the ability to reliably make an observation of truth or falsehood through generalizing the observed function until an observation can reliably be brought into their experience.

Table 1: Components of HCFM based observation.

\section{Applying General Collective Intelligence to the Process of Observation in the Pre-Qualification of Survey Respondents}

A General Collective Intelligence or GCI [2], [3]is a system that combines groups into a single collective intelligence with the potential capacity for general problem solving ability that is vastly greater than that of any individual in the group. To some limit, this capacity can be scaled with the size of the group. In order to reliably have the capacity for complexity that is required for truthful observation, groups must have the capacity to implement whatever GCI functionality that is required to execute that process of observation within a GCI based process, so they have the capacity to apply GCI to increase cognitive capacity to the point they car reliably bring observations into the group's cognitive capacity: 
- The group must implement the features of GCI required to scale capacity for complexity in the specific process of observation.

The specific GCI features required also can be specified as criteria that any observation process must have in order to be consistent with having the required subset of features of GCI.

\section{Component of Model}

Functional modeling (problems and solutions)

Functional decomposition

Functional domain bridging

Functional fitness

Functional stability

Functional adaptation

Functional Cooperation

\section{Description}

Components are modeled only by function to remove prejudice for or against any given implementation.

Functional components are decomposed into their most basic functional building blocks for reuse.

Different domains in which different functions are more fit in achieving the same purpose are identified. These domains are bridged by using a set of weights which identify the best function in each domain.

Every functional component is assigned some projected and actual fitness in achieving its function.

For functional components to persist they must display some degree of stability in fitness to function.

For functional components to persist in a changing environment they must have the ability to adapt their function.

One of the most important domains of adaptation is cooperation. Functional components must interact according to the principles of decentralized cooperation in order to maximize outcomes for each. Functions must be prioritized according to the principles of centralized cooperation in order to maximize outcomes globally.

\section{Table 2: Components of GCI process execution.}

In determining the required set of GCI features, the ability to observe the truth accurately by definition must have an impact on observations. By analogy, if an individual has the visual capability to distinguish two points in the distance as separate, and the capacity to understand the question of whether they are separate, then answers above the required threshold of capability and capacity should be positive (the points are separate). Answers below that threshold might be random or might tend to be negative (the points are not separate). That change in coherence then determines the threshold of usability of the data. By generalizing this approach to determine metrics for the capability and capacity of each observer, a matrix of capability and capacity might be defined, assuming that each correct answer is an additional step along each dimension, so that if, as capability and capacity decrease, there is some point at which patterns in responses lose coherence, then results below this threshold might be discarded.

Capacity in general might be defined in terms of being able to understand and accurately respond to a set of questions. Capability in general might be defined in terms of non-capacity related attributes required to accurately respond. These might include personality attributes, beliefs, cognitive biases, or any other inclinations that might exist. For example, if an individual indicates they do refuse to complete a certain task under any circumstance, then asking that individual "should that task be done?" might be likely to produce a biased answer. To avoid introducing bias through this consideration of capability, the capabilities required for each answer must be defined through statistical analysis of cases in which that particular answer led or is projected to lead to optimal outcomes in terms of well-being. For example if the answers to the question "Have you observed bravery to be useful in war?" are "Yes" or "No", then getting answers from war mongers might be biased to see war as positive, and so might 
be biased to answer "Yes" because they are biased to attribute any positive personal attribute to the desire to engage in war. On the other hand, getting answers from cowards who look to others for their security might be biased to see engaging in war personally as to be avoided regardless of cost, and so might be biased to answer "No" if they are biased to attribute any positive personal attribute to the desire to avoid war. Of course, if they are self-hating and acknowledge or even exaggerate their cowardice rather than simply not being cowards, then they might be biased to answer "Yes". In either case, their answer will be colored by their own bias.

Rather than defining truth from the perspective of any bias, from the functional perspective a process of observation transmits truth [1] if the answer it transmits reliably functions to achieve an outcome. Therefore truth is defined in terms of what observation is proved to reliably function to achieve an outcome. From this perspective one might instead focus on getting answers from parties for whom that truth has functioned to achieve optimal outcomes, such as populations who have successfully resolved each war they've engaged in relatively quickly rather than getting answers from populations who have suffered after having lost, or getting answers from populations that have been devastated by a protracted war or wars, even a war or wars they might have won. In order to make such a complicated assessment, it is necessary to define very simple functional models of the attribute and its function in a given context. For example, there might be a noticeable difference in capability to complete a task like winning a war if bravery is a coherent quality, and if that task requires bravery above a given threshold, when one lacks bravery at or above that threshold.

In the specific case of the experiment to validate the potential for GCI to significantly increase a group's general problem solving ability, which this paper was initially conceived to be of guidance in, the theory of GCI suggested that observing the truth of this claim was not reliably achievable without GCI. And so implementation of a small subset of GCI would be required to validate a small portion of this question, and that validation process could be used to build a larger set of GCI that could then validate a larger portion of that question, in an iterative cycle that eventually answers the larger question. This experiment was intended to begin with the subset of GCI required to answer the much simpler question of whether GCI has the potential to significantly increase the effectiveness of altruism in one specific case. The end result of the survey is "Yes" or "No" to the question that GCI has the potential to significantly increase the effectiveness of altruism in the specific case mentioned in the survey. The determination of coherence is intended to be an implementation of the GCI feature of adaptive problem-solving. Coherence in the answers at each point in capability-capacity space is expected to be determined by some measure for the departure of data from randomness, so that the experiment can continually adapt by unfocusing on incoherent data and focusing more closely on coherent data.

\section{Conclusion}

In summary in applying HCFM in order to have the capacity to accurately make observations within one's cognitive capacity:

- One must learn to distinguish truth from falsehood by the function of an understanding in one's observations, not in one's beliefs, and not in one's assumptions.

- One must learn to expand one's ability to observe by generalizing until an observation can reliably be brought into one's experience.

These can be specified as criteria that any observation process must have in order to be consistent with the HCFM methodology:

And in applying GCI in order to have the capacity to increase cognitive capacity to bring observations into one's cognitive capacity: 
- One must implement the features of GCI required to scale capacity for complexity in the specific process of observation.

These also can be specified as criteria that any observation process must have in order to be consistent with having the required subset of features of GCI.

\section{References}

[1] Williams, A. E. (2020, April 27). A Mathematical Model for Identifying Truth in Observations Made within Individual Human Self-Awareness. https://doi.org/10.31730/osf.io/4nsgk

[2] Williams, Andy E. “A Model for General Collective Intelligence.” AfricArXiv, 30 Apr. 2020. Web. [3] The Relationship Between Collective Intelligence and One Model of General Collective Intelligence, Andy E. Williams, Computational Collective Intelligence, 11th International Conference, ICCCI 2019, Hendaye, France, September 4-6, 2019, Proceedings, Part II, Pages 589-600 